\title{
Meta
}

Journal des traducteurs

Translators' Journal

\section{Champs, clôtures et domaines : des langues de spécialités à la culture scientifique}

\section{François Gaudin}

Volume 40, numéro 2, juin 1995

Usages sociaux des termes : théories et terrains

URI : https://id.erudit.org/iderudit/002821ar

DOI : https://doi.org/10.7202/002821ar

Aller au sommaire du numéro

Éditeur(s)

Les Presses de l'Université de Montréal

ISSN

0026-0452 (imprimé)

1492-1421 (numérique)

Découvrir la revue

Citer cet article

Gaudin, F. (1995). Champs, clôtures et domaines : des langues de spécialités à la culture scientifique. Meta, 40(2), 229-237. https://doi.org/10.7202/002821ar
Résumé de l'article

L'examen critique de la notion de langue de spécialité révèle l'ignorance des différentes logiques linguistiques, fondées sur des oppositions culturelles, donc historiques, qui caractérisent les disciplines. Aujourd'hui, les nouvelles sciences doivent être envisagées non comme des domaines, mais plutôt comme des réseaux de noeuds, les échanges primant sur les particularismes. L'interdisciplinarité suscite des besoins d'échanges qui ne sont pas étrangers aux problèmes posés par la transmission et la mise en culture des connaissantes Cette perspective rejoint les préoccupations des terminologues du fait de l'importance que prend la qualité de l'équipement linguistique des sciences et techniques de demain. 


\title{
CHAMPS, CLÔTURES ET DOMAINES: DES LANGUES DE SPÉCIALITÉS À LA CULTURE SCIENTIFIQUE'
}

FRANCCOIS GAUIDIN

Universiric de Romen. Roulen. France

\begin{abstract}
Résumé
L.'examen critique de la notion de langue de spécialité révele l'ignorance des différentes logiques linguistiques, fonde's sur des oppositions culturelles, done historiques, qui

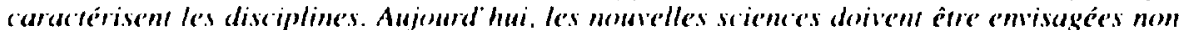
comme de's domaines. mosis plutist comme de's re'seaur de natuds. le's échanges primant su les particularismes.

L'interdisciplinarité suscite de's besonins d'échanges qui ne som pas érrangers aux

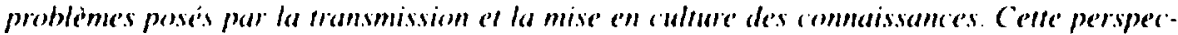
tive rejoint les prencoupations des terminologues du fait de l' importancer que prend la qualine

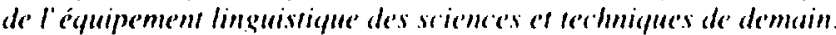

\section{PREAMBULE}

La critique de la notion de languc de spécialité a été faite à plusieurs occasions (Gaudin et Assal 1991: Gambier 1988; Gaudin 1993a). Il est clair qu'il ne s'agit pas d'un concept. mais d'une notion de sens commun qui peut constituer un obstacle, si l'on se pose des questions d'ordre épistémologique. ("est pourquoi nous avons proposé de lui substituer la notion d'épistémé. popularisée par Michel Foucault (Gaudin 1993a). Cependant, dans la pratique quotidienne, la notion de langlue de spécialifé est utile, du fait notamment de son efficacilé documentaire, bien qu'elle ne possède qu une valeur empirique. Il est d’ailleurs intéressant de voir comment Rostislav Kocourek défend la notion de façon très argumentée (1991: 13-25) et la définit comme "une variété de langue à dominante cognitiv' 's (1991: 42).

La difficulté réside justement dans cette notion de variété, qui appelle un dénombrement. Et l'on sait déjà quelles difficultés pose le dénombrement des langues, alors le dénombrement de variétés... Pour terminer sur ce point, laissons un instant la parole à Michel Serres:

La culture ne se partage pas, les sciences ne se divisent pas, rien de tout cela ne se classe. Une dernière preuve? Tentez de définir chacune de ces instances. Le mot le dit. si vous pouvez couper. diviser, partager, vous pouvez définir. Or vous ne pouvez pas $|\ldots|$ Seul le pouvoir découpe le savoir. À l'état paisible, il est dense. (1980): 153-1.54)

Soyons paisibles et acceptons donc la densité multiforme du savoir. C'est ce qui en fait une culture. Nous y reviendrons.

\section{EN ROUTE}

Assurément, ce problème de l'appellation langue de spécialité n'est pas important: il ne serait aisément visible qu'à condition qu'un corps de doctrine soit constitué. Or. les recherches menées autour des pratiques langagières en situation de travail el de transmission 
de savoir en sont encore, me semble-t-il, à un stade largement préscientifique. Sur ce point, il me serait difficile de rallier loptimisme de Jean-Claude Boulanger et Marie-Claude L'Homme lorsqu'ils affirment:

La terminologic a maintenant atteint le stade de la maturite certaine qui en fait lune des composantes essenticlles des sciences du langage |... F Flle possede maintenant les indispensables fondements thériques et méthodologiques qui personnalisent une science (1991:23)

Qu'ils nous permettent de douter et de chercher encore des signes nets de l'autonomie épistémologique de la terminologie, qui constitue assurément le noyau dur des langues de spécialités. Ce scepticisme. Ioin d'indiquer un motif de dédain, me paraît encourageant: il existe peut-être là quelques problèmes à résoudre et, au-delà. des besoins sociaux auxquels répondre.

Si les linguistes peuvent se rendre utiles, cette utilité ne doit pas être envisagée sous le seul aspect de la recherche appliquée. C"est là le risque du "pilotage par l'aval». La vocation des universités ne saurait se limiter à la prestation de services, fussent-ils excellents. Cest dire que le travail sur des terrains, tout en étant indispensable, doit rester lié a une réflexion désintéressée et rigoureuse. Or, pour travailler correctement sur les pratiques langagières dans des sphères d activité, pour micux comprendre le monde qui se dit autour de nous, il nous faudrait être en mesure de décrire les différentes logiques linguistiques mises en ceuvre dans les disciplines, dams les corps de métier. Mais nous ne savons presque rien des traditions dénominatives, des histoires lexicales et conceptuelles, des idéologies linguistiques qui conditionnent la mise en mots des connaissances produites.

\section{I.A I.ANG;UE. INTKOUVABIE:}

II existe là une diversité très riche, se dit-on de façon intuitive. et cette diversité n'est sans doute pas étrangère aux raisons qui poussent à parler de langues de spécicalités. Mais il nous manque sur la question des travaux comparatifs qui s'attachent a l'aspect le plus strictement linguistique des vocabulaires et des usiges. C"est pourquoi la notion de langure de spécialité semble indéfinissable. Et même quand ils revendiquent une aethnolinguistique de l'écrit". les chercheurs avouent être aloin d'une définition précise de l'objet "langue de spécialité". "kextes spécialisés" ou "discours spécifique" (Spillner 1992: 42).

Ce qui guette le chercheur. c'est alors l'inévitable reconduction d'un partage posé d'avance qui $n$ 'autorise, somme toute, que des suppositions: "il est à supposer qu'une langue de spécialité peut se distinguer de la langue commune par des traits spécifiques situés à tous les niveaux de la languen (Spillner 1992: 45). On peut certes le supposer...

Autre problème récurrent, la notion de langue de spécialité est souvent définie par rapport à une autre notion, aussi floue. On ne fait alors que noyer le poisson conceptuel en le déposant dans un bassin voisin: "l'analyse linguistique des langues de spécialité me peut déduire el définir les langues de spécialité que de la communication spécialisée." (Spillner 1992: 46). Mais quand la communication est-elle spécialisée"? Et, en recourant it la notion fourre-tout de communication. peut-on progresser?

Il serait inutile de multiplier les exemples. On peut renvoyer le problène it la définition d'une "communication spécialisee"e comme le fait ici Bernard Spillner. On peut également définir la langue de spécialité "par le domaine où elle est employée», comme le font Bodo Mïller (cité dans Kocourek 1991 : 25) ou I'ISO); c'est alors domaine qui pone probleme. II n'est qu'à lire la détinition qu’en donne I'ISO): "domaine: partie du savoir dom les limites sont définies selon un point de vue particulier". Qu est-ce qu un "point de vue particulier". sinon une façon de formuler l'embarras?" Quelles que soient la stratégie et l'orientation du travail, on se trouve toujours face à une notion de sens commun. Comme on le voit, il est difficile d'avancer. 


\section{LE HABÉLISME: SPECIALISF:}

Il est difficile de définir le concept. mais en même temps ces «langues introuvables» ont tendance à voir leur nombre se multiplier. On craint que les sciences et les techniques ne conduisent à un babélisme généralisé. C"est le problème classique du partage de la science en disciplines, qui a suscité maintes entreprises classificatoires. Or. ce à quoi on assiste. c'est à un renversement de tendances: ce qui féconde les sciences aujourd hui ce n'est plus un repli des savoirs sur eux-mêmes, le labour sérieux d'un lopin conquis de haute lutte. c'est au contraire l'échange et le métissage. Comme lè résume Michel Serres: "la science n'est pas un contenu, mais un mode de circulation" (1972: 154).

Il s'agit là d'une évolution historique qui doit conduire à penser autrement. L'attitude traditionnelle procède d'une métaphore jadis séduisante, aujourd hui gênante: celle du domaine. En fait, les nouvelles sciences doivent être envisagées non comme des domaines, mais plutôt comme des réseaux de noruds: "les domaines singuliers deviennent des échangeurs, de concepts, de méthodes, de modèles» (Serres 1972: 6.5). Les problématiques actuelles naissent donc de contacts interdisciplinaires. d'échanges de concepts et de mises en cornmun de compétences.

Pour illustrer ceci. examinons quelques libellés d'intitulés de laboratoires pris dans une brochure du CNRS2: "Biologie des populations d'Helminthes Parasires", "Génétique et pathologie moléculaires". «Régulation métabolique et génétique des biosynthèses». "Chimie du solide et inorganique moléculaire". "Magnétodynamique des liquides, applications à la métallurgiè. Ces quelques titres en témoignent, la recherche d’aujourd hui est celle des contacts disciplinaires è de la centration sur des objets d’étude.

\section{LA CIRCUL.ATION DES MOTS ET SAVOIRS}

Mais cette ouverture sur les autres disciplines et l'importance prise par les applications ne viennent pas nécessairement contredire l'existence de vocabulaires particuliers. Bien au contraire: si l'on souligne les jonctions, c'est bien que les frontières sont sensibles. On peut poser comme hypothèse qu il est peut-être des langues du savoir, des traditions linguistiques reflétant des histoires locales de la pensée, ou des aventures régionales de la transformation du monde. En ce cas. il ne faut pas perdre de vue que "la communauté savante est désormais polyglotte» (Serres 1972: 27). En effet, la circulation horizontale des savoirs et des modèles, donc des mots. est devenue une nécessité. Et les linguistes peuvent peut-être contribuer à son amélioration.

Il en va du langage comme du savoir: les échanges priment sur les particularismes. Les mécanismes identitaires sont toujours remis en aruvre dans des procès infiniment rejoués: on n'en finit jamais d'être soi-même et de s'affirmer dans les échanges... Et de ce point de vue, le jargon marque à la fois la clôture sur un savoir non partagé et l'appartenance à un corps professionnel, à une école, à un réseau. C'est pourquoi le chercheur en position de vulgarisation éprouve fréquemment une insécurité linguistique: c'est qu'il doit renoncer au jargon. Et si le jargon possède donc une fonction scciale indéniable, cette fonction identitaire ne justifie pas que l'on assigne à chaque collectivité une langue de spécialité.

\section{LA MANIF (LASSIFICATOIRE:}

La division en langues de spécialité constitue donc un pur artefact. Elle peut aider à répartir des documents: elle peut satisfaire des manies: elle peut $s$ "inscrire dans une épistémologie à tendance délirante (Auguste Comte est tout ensemble un grand néologue et un grand classificateur); la connaissance des faits de langue concernés n'en est pas avancée. 
Partons d'une attitude raisonnable. On peut supposer, comme l'imagine Allal Assal (1992: 26), que la biologie et les mathématiques se distinguent par des langues (LSP) spécifiques. Certes. Il suffit d'être bachelier pour en avoir le sentiment: on parle différemment des logarithmes et de génie génétique. Il s'agit simplement de ne pas multiplier à linfini le nombre de "LSP" au gré du découpage institutionnel: va-t-on parler d'une nouvelle LSP des qu un champ de recherche accede au rang de section du CNRS ou du CNU"? que serait, par exemple, une I.SP des "sciences cognitives"), des "sciences pour l"ingénieur". de la "mécanique des fluides", des asciences de l'environnements"? Le découpage en langues de spécialités devria-1-il suivre les redécoupages institutionnels. les mises à jour des classilications documentaires, des banques de données?

\section{F(CRITURE FIT DIAI.O(;IF:}

Indiquons une autre limite. La problématique des langues de spécialités se limite souvent à la seule dimension écrite. Or, $n$ 'oublions pas la différence essentielle qui sépare l'oral et l'écrit. En privilégiant excessivement l'écrit, la linguistique s'est par moments attardée dans des impasses; que l'on en retienne au moins la leçon: il faut aussi écouter comment les gens parlent. et se parlent, pour comprendre comment ils usent des mots. comment ils en créent. pourquoi ils s'y heurtent.

Dans le travail collectif, et quelle que soit l'institution qui l'organise. la parole joue un rôle essentiel et premier. ("est à l"oril, et heureusement, que l'on discute, que l'on échange ses perplexités, que lon «ferraille» sur des points litigieux. Il n'est pas rare que ce soit en en parlant que l'on trouve la solution d'une difficulté. Ainsi, raisonner sur le seul écrit induit en erreur et conduit à méconnaître le fait que certaines communautés scientifiques. comme l'affirme Benoît Jurdant, travaillent pour l'essentiel a l'oral, par exemple. les physiciens des particules. El c'est là un aspect trop méconnu du travail scientifique. Toutefois. concernant l'oral. la prudence s'impose car très peu de travaux lui sont consacrés. Il faudrait étudier de très près less communications réelles en situation de travail. en prenant en compte la diversité des échanges, des profils et des compétences.

Découvrir, parler, ceest tout un: la pensée inventive n'est pas muette. Elle n'est pas l'affaire d'hommes et de femmes isolés: elle est par nature sociale, socialisée. Ici encore. isoler, cest trancher. Que l'on s'intéresse au niveau linguistique ou au niveau cognitif. dans les deux cas. le dialogue est premier. Le dialogisme dont parle Bakhtiner. présent dans les textes. l'est aussi dans toute cruvre collective de conceptualisation. Et cette dimension est essentielle dans la mesure où elle permet de réviser des postulats informulés.

\section{DOMAINES F.T CLOOTIRES}

Cess postulats tiennent notamment à la clôture des disciplines. Cette relative clôture existe dans les faits, dans les objets étudiess; mais il y a un risque à étendre ce partage, lié finalement à la division du travail el a la spécialisation, aux concepts, aux mots et aux pratiques langagières. Nous avons besoin de fermer les portes de nos bureaux. de nos laboratoires, pour travailler en paix. Le travail sépare. isole. C'est pourquoi nous morcelons le monde. les atomes, la matière, les mouvements, les idées, les sens, les sons...

Mais la langue. la pensée, les mots ne connaissent pas de frontière. En travaillant. nous parlons, avec nos collègues, parfois seuls, souvent au téléphone; nous écrivons pour fixer des idées ou transmeltre des résultats. Bref, la langue est partout. Done, les mots. Et avec les mots, les idées, les habitudes et les schémas de pensée qui les accompagnent. leur mémoire collective et intime. Et cest dans celte circulation que se refaçonnent nos façons de dire et de penser le monde. car ula conceptualisation a pour matériau verbal des schèmes de sens préexistants, qui se transforment pour parler d'autre chose's (Schlanger 1988: $8(0)$. 


\section{A TRAVERS CHAMPS}

C'est pourquoi il faut bien voir que les idées nouvelles ne naissent pas dans des enclos, à l'intérieur de propriétés privées, mais plutôt dans des champs: les idées ont ceci de commun avec le gibier qu'elles n'appartiennent jamais au propriétaire du terrain mais à celui qui les piste, qui les fait travailler et parcourir de nouveaux espaces, faisant montre d'une longue patience. On "poursuit" son idée. on traque sa propre pensée. En cherchant le mot juste, on peautine sa conceptualisation: filer la métaphore évoque la filature. Parler avec des mots nouveaux, penser de façon inhabituelle, c'est aussi traquer des programmes de sens, questionner des analogies. Les philosophes des sciences parlent pour ces phénomènes de "concepts nomades» (Stengers 1987). Leurs travaux sont passionnants mais obligent le linguiste à s'interroger: se situe-t-on au niveau conceptuel, cognitif, ou linguistique?

Nous parlions d idées, mais les idées ne se rencontrent pas: nous ne rencontrons que des signes. Ce que nous disions du nomadisme des idées vaut pour les mols. Ils circulent. En tout cas. les mots yui servent à travailler ensemble sur des objets particuliers, au sein d'institutions particulières. en respectant des partages de compétences garantis par des instances nationales et disciplinaires. ces mots ne connaissent pas de frontières naturelles. à l'intérieur de ces grands ensembles llous que sont les langues. Et les mots. les langues. sont des formations culturelles.

Il n'existe que des commumantés. aux contours plus ou moins définissables, qui laissent le linguiste, ou le sociolinguiste. bien démuni. En la matière, la misère de la lexicographie n a guère été secounue par la linguistique, fût-elle sociale: on continue à raisonner en qualifiant les mots de "techniques, médicaux. populaires ou argotiques». comme dit la chanson. Évidemment, la mise en lumière de ces lacunes, de ces doutes, ouvre un espace de recherche. Cel espace part du travail et s'ouvre sur la culture. Expliquons-nous.

L'étude des usages linguistiques dans des milieux professionnels se cale le plus souvent sur le milieu de travail. Mais les étiquettes utilisées pour découper des formations discursives - telle pourrait être l'ambition - sont purement institutionnelles et ne correspondent ni à des découpages consciemment effectués par des individus, ni à des conditions de production de discours.

II y a. certes, de grandes oppositions culturelles. donc historiques, qui permettent aux locuteurs de se dire chimiste, biologiste ou physicien. Mais dès que l'on descend à un niveau plus fin. celui évoqué dans la section sur la manie classificatoire. les dénominations deviennent moins pertinentes: la chimie des solides, la stochastique, la pragmatique, la mécanique des fluides. etc.. ne constituent pas des secteurs culturels qui puissent légitimement fonder un découpage utile au linguiste. Il s'agit là de découpages institutionnels, qui permettent le partage et la répartition du travail... et des crédits, mais dont il nous paraît nécessaire de se détacher pour s'attacher aux particularifés proprement linguistiques.

\section{DU PLAN DU SAVOIR Ȧ L.'FSPACE ( ULTUREI.}

Cet aspect-là débouche directement sur une conception plus culturelle de la science. tant dans son unité que dans son insertion dans la culture commune. C'est en travaillant sur les mots, sur les formes, que l'on peut étudier l'aspect culturel des parlers scientifíques, ce qu'ils ont en commun. même s'ils l'articulent un peu différemment aux objets qu'ils étudient.

Mais également, il est important de mettre en lumière l'importance des échanges entre culture quotidienne el termes. Les termes des sciences, des techniques, etc., nous offrent des schèmes de pensée qui suscitent des métaphores quotidiennes précieuses. Songeons à cette influence réciproque que la chimie nous a permis de nommer osmose: 
aux reproductions strictement conformes à leurs modèles que nous appelons, après les biologistes, des clome's. Mais les échanges vont dans les deux sens: notions quotidiennes et concepts ne sont pass séparés. Comme l'indique la morphologie. la percolation du physicien et le percolate'ur du cafetier possèdent un principe commun?.

Or l'accent est trop souvent mis sur la désignation (aux dépens de la signilication) et la spécialisation des termes. ce yui tend à gommer leur importance culturelle. Pourtant. nos conversations quotidiennes sont pleines d'équations, de vitamines. de virus: nous parlons sans étonnement de temps réel, nous prenons lat tangente, nous souhaitons vivre en symbiose. à lout le moins en symergié, nous nous méfions de notre inconsciem... L'histoire de l'immunologice est l'histoire d'une mélaphore: l'immonité parlementaire, sens historiquement premier, se révèle finalement hien moins vulnérable que l'immunitó que nous procurent nos inticorps...

Cette intrication lexicale peut être un facteur de umise en culture» des connaissances, tandis que l'usage de noms trop "cryptiques» est facteur d’incompréhension. Par ailleurs, le travail sur les formes permet également de typifier les traditions dénominatives selon, par exemples, le plus ou moins grand recours aux formes gréco-latines, aux emprunts, aux métaphores. selon les concurrences synonymiques héritées de l'histoire...

Ainsi. cest pour des raisons largement morphologiques que le vocabulaire de la médecine joue un rôle d'écran dans lés interactions entre médecin et patient. Le recours au jardin des racines gréco-latines et a des supplétismes cutilisation de formes concurrentes, souvent grecques ou lattines, pour remplacer des mots français) pour dénommer des concepts est traditionnel el e justifie par la recherche de la concision et de l'univocité. Par rapport à scumner. tomodensitométlé est très descriptif el motivé. pour qui peut le rattacher à une forme grecyue "lomos» ou l'insérer dans un paradigme comprenant tomographice et densitumetre'. (iageons que l'usager moyen est rarement dans ce cas de figure: il aura plutôt repéré dans les médias la forme anglaise scammer.

En fait, si ce recours aux ressources gréco-latines a pu se justifier, aujourd hui il n'est plus adapté ni aux habitudes linguistiques de nos contemporains, ni au rôle social que remplit la médecine. Á l'aube du XXI" siècle. cette tradition dénominative, qui a rendu de précieux services, constitue indéniablement un ficteur de dysfonctionnement dans les interactions verbales et apparaît comme un jargon à fonction de clôture.

Si de telles études contrastives peuvent être intéressintes, c'est bien sûr pour les besoins de l'enseignement de la traduction, de la rédaction technique et scientifique, mais c'est aussi pour qu'il soit un jour possible aux linguistes de jouer un rôle de conseil en matière d'équipement linguistique auprès des chercheurs. des techniciens, des rédacteurs.

\section{DES QUESTIONS GILOTTOPOLJTIQURS}

Nous touchons là un point de jonction entre la nécessité de transmission des commaissances. par leur mise en culture. el l'impératif, bien connu des terminologues, de

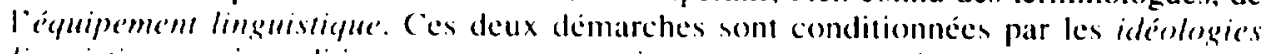
linguistigues qui conditionnent toute entreprise peu ou prou aménagementiste. Qu est-ce à dire?

Less idéologiess linquistiques jouent fortement sur l'équipement lexical des disciplines. Ce peut être un élément de contrasté. La réputation d'un Benoît Mandelbrot, en mathématiques, celle d'un René Thom. en physique, jouent très certainement un rôle important dans l'attitude des mathématiciens et des physiciens par rapport aux choix dexpression qui les caractérisent. Thom et Mandelbrot utilisent une terminologie largement française. et francisée, yu'ils diffusent à travers leurs travaux. là où un chimiste aussi prestigieux que Jean-Marie I.ehn's par exemple, cherche plutôt à privilégier des 
dénominations de concepts présentant une grande similitude de signifiants d'une langue à l'autre. Ceci n'étant sans rapport avec sa conviction, souvent réaffirmée ${ }^{9}$, que la science est indépendante des langues.

Ces attitudes, spontanées de la part des locuteurs, sont à replacer dans le cadre du rapport de forces conditionnant la circulation des énoncés. On ne peut intervenir correctement que si l'on décrit préalablement la situation du marché linguistique.

Donnons quelques pistes: Quel est le marché linguistique de telle discipline? Quelle part les chercheurs accordent-ils à la diffusion des résultats, à la transmission des connaissances, à la vulgarisation? Quelle est l’influence de l'anglais? Quelle est la place de l'industric nationale dans le développement de tel secteur? Quelle est la place de la recherche française sur le plan international?

Ce sont là des problèmes de ghottopolitique (Guespin 1985) qui commandent une approche sociolinguistique, et sont complémentaires de l'étude des besoins dénominatifs: où a-t-on besoin de mots français'? comment peuvent-ils être acceptés au mieux? quels canaux de circulation emprunter?

Et ce n est pas un hasard si notre réflexion sur la dimension culturelle du travail scientifique nous fait rencontrer des problèmes d'aménagement. En effet, chercher à insérer des unités dans un système linguistique nécessite une connaissance des valeurs sémantiques et culturelles constituant le systìme linguistique sur lequel on se propose d'intervenir. Or pour parvenir à celte connaissance, il faut dabord une description des pratiques langagières réelles.

Il est donc important de tenir ensemble les deux bouts de la chaîne, théorie et pratique. Mais plus encore. ce qui importe. c'est de ne pas limiter l"étude de la terminologie à des aspects trop restrictifs. Considérée dans son rapport aux connaissances, aux pratiques. aux milieux professionnels, aux conditions éditologiques de sa circulation, replacé au sein des enjeux traversant le marché des langues. la terminologie se révèle comme un champ nécessitant une approche pluridisciplinaire. $\dot{A}$ sa façon. il s'agit d'une métadiscipline fondée sur une approche linguistique.

\section{OBSFRVER DES SIINNES}

Mais pour ce faire, les seuls faits que nous puissions observer sont les signes, principalement linguistiques mais non exclusivement, que nous rencontrons dans les textes. C'est pourquoi la méthode employée doit faire largement place aux données linguistiques.

Tout d'abord. cela suppose de partir des textes et non d'unités baptisées aconcepts» dont le linguiste ne sait rien. On sait que dans La linguistique de la langue saramte. un vocable est dit terme du fait de "l’existence d"une définition spécialisée" (Kocourek 1991: 1511. C'est donc la définition qui fait le terme, donc le concept. Lioptique choisie est référentielle; ce qui a pour conséquence la multiplication des homonymes et l'engorgement des hases de données. Par exemple, on définira différemment le charbon actif selon qu'il est employé dans l'industrie du tabac ou l'épuration des caux.

Plus intéressantes sont les orientations sémantiques, par exemple celle proposée par Pierre Lerat (1988) qui, basée sur le repérage des cooccurrents, tente de cerner la valeur d'une unité par sés relations lexicales. On peut, dans cette voie, mettre en rapport les différents usages d'un même terme. en faisant apparaître ce qui les distingue: on évitera ainsi de décrire différemment le terme somificatelur selon que son «action typique» est de dissoudre des précipités ou de laver des pipettes. cas dans lequel on l'appelle aussi lavelu à ultrasons. Il en va de même des autres rubriques permettant de consigner les objets, agents et applications typiques. 
II s'agit donc d'envisager le terme comme centre d’un schéma tout uniment lexical et cognitif et. dans le traitement dess polysèmes, de ne pas éclater indûment des unités utilisées massivement avece un "programme de sens" unique lo. les différences résultant de l'ajustement référentièl.

En fiat. on ne peut prendre en compte la dimension culturelle, et donc historique, des signes sans réintégrer leur maissance polvsímique'. Il n'y a qu' en réintégrant cette dimension que lon peut espérer faciliter la communication du savoir. Satvoir ce qu'étiquettent les. mots ne suffit pas: pour que les mots aient un sens, il faut pouvoir comprendre ce qu'ils disent de la réalité en la dénommant.

Comme ke dit Stella Baruk dans l'introduction de son Dictiommaire de Mathématiques.s. "pour que les mots savants" premnent place à côté des autres, il faut les 'raccrocher' a la fois entre cux et a d'autres, yui sont dans la langue naturelle" (1992: 20). Là où l'on présente les termes comme étiquetant de façon arbitraire des concepts. il s'agit au contraire d'insérer les termes dans le système de valeurs que constitue la langue. l.e savoir qu'ils implicitent peut. parfois. être pré-construit si l'on sappuie sur ce que dit le mot dans d'autres usages, ou ce que permet de deviner la forme. Le percolatere peut aider a comprendre ce quest la percolation: dans orthogomal, il y a ortho- et -gemal. que lion retrouve dans orfhoggraphe' el diagromed.

C"est une des apories de la stratégie définitoire que de rédiger des adéfinitions creuses parce que les mots ne sont pas enracinés dans du senss (Baruk 1992: 20). Pour permettre laccès it den concepts, il faut, quand faire se peut, relier l'acception particulière qui permet de construire le concept alu signifié.

Pour comprendre modisateur. il fatut. avant de décrire le référent, expliquer ee qu'il modère:

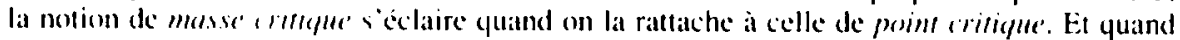
les dénominations des concepts somt arbitraires et coupées des signifiés, il faut le souligner

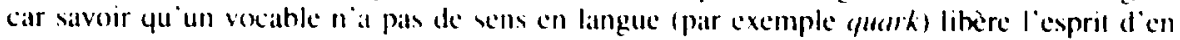
chercher unt. (ciatudin 1994: 58)

De ce point de vue, les dictionnaires scientifiques constituent un bon observatoire des différentes stratégies mises en acuvre pour mettre en mots le savoir 'I. Animées le plus souvent d'un même souci, d'une méme générosité. elles s iavèrent inégalement opératoires. Pourquoi?

Entre autres catuses, parce que de nombreux scientifiques éprouvent des difficultés à se priver des facilités que leur procure l'usige de leur jargon, des mots de leur tribu. Cess difficultés peuvent avoir des raisons idkóologiques, plus ou moins conscientes'?: mais pour lessentiel, il sagit la de problemes linguistiques. Rédiger. reformuler, mettre en place der réseaux de signes nouveaux, tour cela suppose une lechnique et nécessite une information éclairée sur ce yu ess la langue, sur ce qui facilite l'intercompréhension et sur les moyens de l'optimiser.

Voilat une tâche à laquelle les linguistes peuvent concourir. Mais auparavant, leur premier devoir est de comprendre le suful de la sciemece. des disciplines et des systèmes de connaissances du temps dont ils veulent ćludier les pratiques langagières. En effet, que sera l'étude du vocahulatire scientifique, si l'on ne sait ni qui utilise ce vocabulaire, ni de quelle science il parle"? Matis cest unce autre histoire... 
Notes

1. Ce texte constitue la version définitive du texte d'une communication présentéce lors de la $4^{c}$ journée ERL.A. (il.AT a Brest en 1993 el publice avec de nombreuses erreurs dans les actes.

2. Ces intitulés concernent des laboratoires dont des membres ont été médaillés en 1992 par le CNRS.

3. Conseil Vational des linversites.

4. Devenue's depuis peu "neuro-sciences intégratives"..

5 Qui ont remplace l' accolongè" discipline plus traditionnelle et conforne au découpage positivisle.

6. (f) Bakhtine, Mikhail, 1977.

7. Sur ce point, vour Giludin 1992.

8. Prix Nobel de chimic.

9. Par exemple lors de l'ouverture du forum "Quelles langues pour la science?".

10. Pour lapport de la praxematique a la terminologie, on pourra consulter le demier chapitre de (jaudin. $194.3 \mathrm{t}$.

11. Cf Ciaudin, 1902.

12. Sur ce puint, / Kunth. 1992.

\section{RFFERFNC FS}

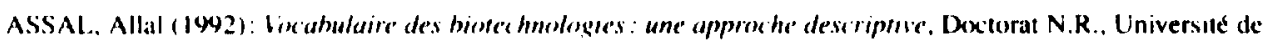
Rouen. 317 p.

BAKHTINE. Mikhall (1977): Le marvame a la philosophie die langage. Essai d"application de la mithode

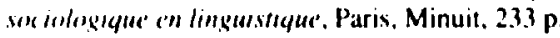

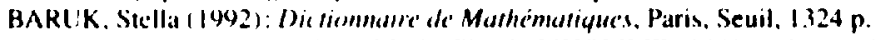

BO(11.AN(il:R. Jean-( "laude et Marie-("laude L.HOMME (1991): “Len technolectes dans la pratique dictionnarique genérale: quelques frangments d une culture "., Mefo, vol, $36, n^{\circ} 1, \mathrm{pp}, 23-40$ ).

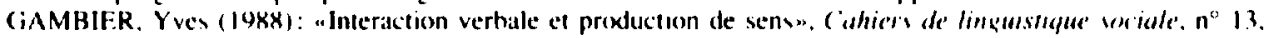
Rouen, I miverste de Rouen. $192 \mathrm{p}$.

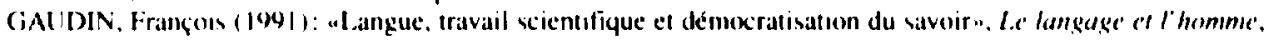
vol. Xxvi, n" 2-3. Bruxelles, éd. Instifut Lihre Marie Haps, pp. 129-139.

(iAIIDIN. François (1992): “Terminologie el démex ratisalion du savoir: a propos de dictuonnaires scientificuess.

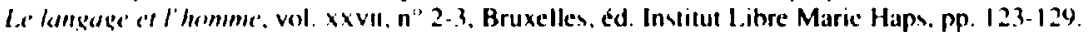

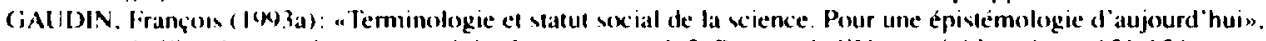

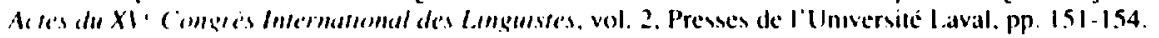

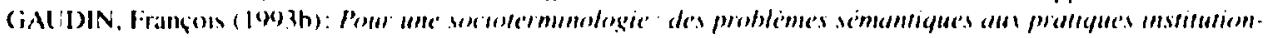
m'lle's. Rouen. Publicallons de l'Inivernte de Rouen, $254 \mathrm{p}$.

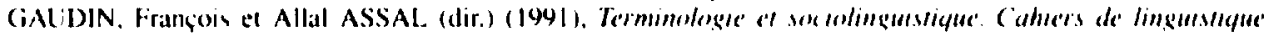
soriale, n" 18. Rouen. Universite de Rouen, $21.3 \mathrm{p}$.

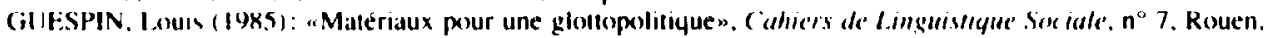
Publecations de l'o niversite de Roucn. pp. 13-32.

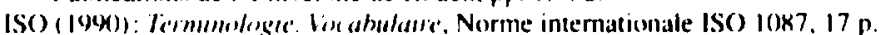

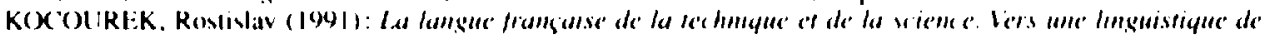

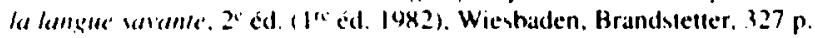

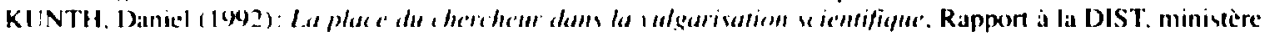
de la Recherche el de l'Expace. $8.3 \mathrm{p}$.

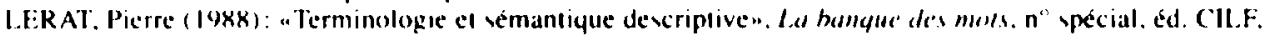
Pp. II-., I).

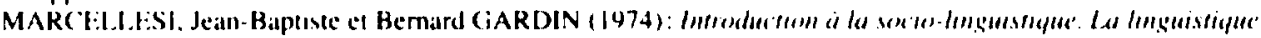
whisle. I arouse. $26.3 p$

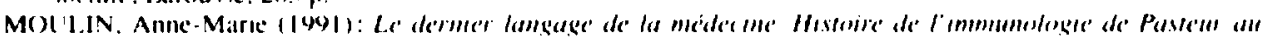
Sirla. Parm. PIIF, 447 p.

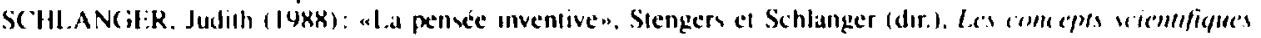

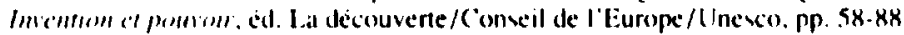

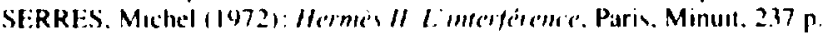

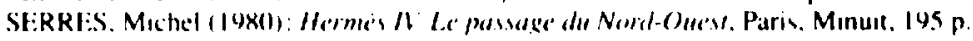

SPIL.INER. Bernard (|W92): "Texte medicaux français et allemands. Contribution a une conparaison interlan-

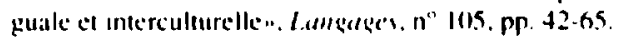

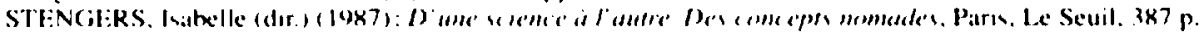

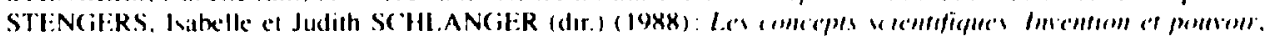

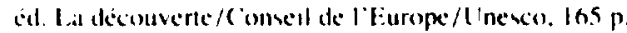

